\title{
Teoría instruccional y tecnología para el nuevo paradigma de la educación
}

\section{Instructional Theory and Technology for the New Paradigm of Education}

\author{
Charles M. Reigeluth \\ reigelut@indiana.edu \\ Universidad de Indiana \\ Traducción al español de Nora Lizenberg \\ nlizenberg@gmail.com \\ Universidad Tecnológica Nacional, Argentina \\ Adaptación de Miguel Zapata-Ros, revisada por el autor \\ mzapata@um.es \\ Universidad de Alcalá
}

\begin{abstract}
Resumen
En este artículo se describe la teoría instruccional como base y apoyo a la educación postindustrial y a los sistemas de formación - a los sistemas personalizados y que están centrados en el alumno, en los que el progreso del estudiante se basa en el aprendizaje más que en una organización basada en el tiempo. El artículo describe los métodos universales de enseñanza, los métodos situacionales, también describe las ideas centrales del nuevo paradigma post-industrial de instrucción, la importancia y los problemas con la educación basada en la tarea. Los aborda desde la visión de una teoría de la instrucción para la educación y formación post-industrial, así como los papeles que pueden jugar el profesor, el alumno y la tecnología en el nuevo paradigma.
\end{abstract}

\section{Palabras clave}

Teoría instruccional, paradigma post-industrial, educación basada en tareas.

\begin{abstract}
This article describes instructional theory that supports post-industrial education and training systems - ones that are customized and learner-centered, in which student progress is based on learning rather than time. The article describes universal methods of instruction, situational methods, core ideas of the post-industrial paradigm of instruction, the importance of and problems with task-based instruction, a vision of an instructional theory for postindustrial education and training, and the roles that may be played by the teacher, the learner, and technology in the new paradigm.
\end{abstract}

\section{Keywords:}

Instructional theory, post-industrial paradigm, task-basd instruction.

Nota: Una parte significativa de este artículo fue publicados en Reigeluth (2011) y Reigeluth (2012). Se incluyen aquí con el permiso de los editores. 


\section{Introducción}

Una de las pocas cosas sobre las cuales prácticamente todos los autores están de acuerdo, tanto en educación como en formación, es que las personas aprenden a ritmos diferentes y tienen necesidades de aprendizaje diferentes. Sin embargo nuestras escuelas, y los programas de formación en general, enseñan una cantidad fija y predeterminada de contenidos en un cierto intervalo de tiempo prestablecido. Inevitablemente, los alumnos más lentos se ven obligados a avanzar antes de haber llegado a dominar el contenido, y así acumulan déficit en su aprendizaje que les dificultará aprender contenidos relacionados en el futuro. Además, los alumnos más rápidos se aburren hasta la frustración y pierden mucho tiempo valioso esperando al grupo para seguir adelante - un despilfarro considerable del talento que nuestras comunidades, empresas y la sociedad necesitan con urgencia. En un sistema que hubiese sido diseñado para maximizar el aprendizaje en verdad no se forzaría a los alumnos a promocionar antes de haber aprendido los contenidos, y no obligaría a los estudiantes más rápidos que esperar al resto de la clase.

Nuestro paradigma actual de educación y capacitación se desarrolló durante la era industrial. En ese momento, no podíamos darnos el lujo de educar o capacitar a todas las personas hasta el nivel superior, y tampoco teníamos esa necesidad. La forma predominante de trabajo era el trabajo manual. De hecho, si educáramos a todos hasta la educación superior, muy pocos estarían dispuestos a trabajar en las líneas de montaje, haciendo tareas mecánicas una y otra vez. Por lo tanto, lo que necesitábamos en la era industrial era un sistema educativo que seleccionara a los estudiantes. Un sistema que separara a los niños que deberían hacer el trabajo manual de los que deberían ser gerentes o profesionales; de este modo, se desaprobaba a los estudiantes "menos brillantes", y se promovía a niveles superiores de educación a los más brillantes. Este es el motivo por el cual nuestras escuelas evalúan por normas más que por criterios para seleccionar a los estudiantes. Lo mismo se aplica a nuestros sistemas de formación. Hay que reconocer que el principal problema de nuestra educación y de nuestros sistemas de formación no son los profesores o los estudiantes, es el sistema - un sistema que está diseñado para clasificar más para la selección que para el aprendizaje (Ver Reigeluth, 1987 , 1994, por ejemplo).

En otros textos, he presentado mis puntos de vista sobre cómo podría ser un sistema educativo post-industrial - un sistema diseñado para maximizar el aprendizaje (Reigeluth, 1987; Reigeluth y Garfinkle, 1994). Con pequeñas adaptaciones, esa visión se podría aplicar también a nuestros sistemas de capacitación. El propósito de este trabajo es describir la teoría instruccional y la tecnología que sostiene a tales sistemas de educación y formación post-industriales. En particular, en este texto:

- $\quad$ Se describirán métodos universales de enseñanza basados en los "principios fundamentales" de Dave Merrill.

- Se discutirá la importancia de adaptar los métodos a situaciones particulares y de resolver la aparente contradicción con los métodos universales.

- Se describirán las "ideas centrales" del paradigma post-industrial de instrucción.

- Se discutirá la importancia y los problemas que presenta el Aprendizaje por Tareas. 
- $\quad$ Se presentará una visión de la enseñanza post-industrial, y será completada con algunas de las más importantes estrategias instruccionales.

- $\quad$ Y por último se describirán los roles que debe desempeñar el maestro, el alumno y la tecnología en el nuevo paradigma.

\section{Métodos universales de instrucción}

M. David Merrill ha propuesto un conjunto de cinco principios instruccionales prescriptivos (o "principios fundamentales") que mejoran la calidad de la enseñanza en todas las situaciones (Merrill, 2007 , 2009 ). Esos principios tienen que ver con la centralidad de la tarea, la activación, la demostración, la aplicación y la integración. Expuestos brevemente, los principios son los siguientes:

Principio de la Centralidad de la Tarea

- La instrucción debe utilizar una estrategia de enseñanza centrada en la tarea.

- La instrucción debe realizarse mediante una progresión de tareas completas cada vez más complejas.

Principio de la Demostración

- La instrucción debe proporcionar una demostración de la habilidad, y que esa demostración sea consistente con el tipo de la componente de la habilidad: de qué clase es, cómo se hace y qué sucede en la ejecución.

- $\quad$ La instrucción debe proporcionar orientaciones que relacionen esa demostración (particular) con aspectos generales de la habilidad.

- La instrucción debe involucrar a los estudiantes en la discusión y la demostración, con otros alumnos de iguales circunstancias de aprendizaje.

- La instrucción debe permitir a los estudiantes observar la demostración a través de los medios que sean apropiados según el contenido.

\section{Principio de Aplicación}

- La instrucción debe lograr que el alumno aplique lo aprendido en consonancia con el tipo de componente de la habilidad: de qué clase es, cómo se hace y qué sucede en la ejecución.

- La instrucción debe proporcionar retroalimentación intrínseca o correctiva.

- $\quad$ La instrucción debe proveer entrenamiento, el cual debe disminuirse gradualmente para realzar la aplicación.

- La instrucción debe involucrar a los estudiantes en la colaboración entre pares. 


\section{Principio de activación}

- La instrucción debe activar en los alumnos estructuras cognitivas relevantes, haciéndoles recordar, describir o demostrar conocimientos o experiencias previas que sean relevantes para él.

- La instrucción debe lograr que los estudiantes compartan sus experiencias anteriores entre ellos.

- La instrucción debe hacer que los estudiantes recuerden o adquieran una estructura para organizar los nuevos conocimientos.

\section{Principio de Integración}

- La instrucción debe integrar los nuevos conocimientos a las estructuras cognitivas de los alumnos, haciéndoles reflexionar, debatir o defender los nuevos conocimientos o habilidades.

- La instrucción debe involucrar a los estudiantes en la crítica entre iguales.

- $\quad$ La instrucción debe lograr que los estudiantes creen, inventen, o exploren formas personales de utilizar su nuevo conocimiento o habilidad.

- $\quad$ La instrucción debe hacer que los estudiantes demuestren públicamente su nuevo conocimiento o habilidad.

Si bien estos principios podrían aplicarse universalmente a todas las situaciones de enseñanza (situaciones que impliquen aprendizaje asistido), los métodos específicos mediante los cuales se implementa cada principio deben variar de una situación a otra para que la instrucción sea de alta calidad (Reigeluth y Carr Chellman, 2009a ). Por ejemplo, para aplicar el principio "La instrucción debe utilizar una estrategia de enseñanza centrada en la tarea" podría ser necesario variar considerablemente la naturaleza de la estrategia de una situación a otra. Del mismo modo, para el principio "La instrucción debe proveer entrenamiento," la naturaleza del entrenamiento podría variar considerablemente de una situación a otra. De manera que vamos a explorar estas variaciones, o esas "situaciones".

\section{Métodos Situados de Instrucción}

Los principios y los métodos de enseñanza pueden describirse con diversos niveles de precisión (Reigeluth y Carr Chellman, 2009b). Por ejemplo, en el nivel menos preciso, Merrill indica que la instrucción debe provenir del entrenamiento. En un nivel de alta precisión, se podría afirmar, "al enseñar un procedimiento, si un alumno se salta un paso durante la ejecución del procedimiento, se debe inducir al alumno hacia la identificación del paso omitido mediante preguntas que lo guíen hasta llegar al reconocimiento de la omisión". Cuando proporcionamos mayor precisión sobre un principio o sobre un método instruccional, por lo general descubrimos que hace falta que éste sea diferente para diferentes situaciones. Reigeluth (1999a) se refirió a los factores contextuales que influyen en los efectos de los métodos como "escenarios". 
El reto para los agentes instruccionales (y por lo tanto para los teóricos instruccionales) es identificar cuáles escenarios son importantes para seleccionar cada método. Además, los métodos pueden combinarse en un "paquete" compuesto de un conjunto de métodos interrelacionados e interdependientes, en cuyo caso tenemos que identificar cuáles son los escenarios relevantes en la selección de cada "paquete" (conjunto de métodos).

Reigeluth y Carr-Chellman (2009a) proponen que existen dos principales tipos de escenarios que requieren conjuntos fundamentalmente diferentes de métodos:

1. Escenarios basados en diferentes enfoques de la enseñanza (medios), tales como:

1.1. Juego de rol (role-playing)

1.2. Sinéctica

1.3. Manejo del aprendizaje

1.4. Instrucción directa

1.5. Discusión

1.6. Resolución de conflictos

1.7. Aprendizaje entre iguales

1.8. Aprendizaje experiencial

1.9. Aprendizaje basado en problemas

1.10. Aprendizaje por simulación

2. Escenarios basados en diferentes resultados de aprendizaje (fines), tales como:

2.1. Conocimiento

2.2. Comprensión

2.3. Aplicación

2.4. Análisis

2.5. Síntesis

2.6. Evaluación

2.7. Desarrollo afectivo

2.8. Aprendizaje Integrado (p. 58)

Los capítulos en las Unidades de 2 y 3 de Reigeluth y Carr-Chellman (2009c) "Libro Verde 3" (Teorías y Modelos de Diseño Instruccional, Volumen III: Construyendo una base de conocimientos en común) describen la "base de conocimientos en común" para nueve de esos conjuntos de métodos.

En el resto de este capítulo, proporciono una visión más holística de lo que el paradigma post-industrial de instrucción podría ser. Empiezo con "ideas centrales", seguido de una visión de lo posible, y, finalmente, abordo los roles de los actores clave para este paradigma de la enseñanza. 


\section{Ideas centrales del Paradigma de Instrucción Post-industrial}

Las siguientes son algunas ideas básicas para el paradigma de instrucción postindustrial. Se presentan como dicotomías para contrastarlas con las ideas centrales que caracterizan el paradigma de instrucción de la era industrial, pero debe entenderse que las dicotomías suelen ser falsas, y que el pensamiento post-industrial se caracteriza más por un planteamiento sumativo del tipo "ambas cosas" que por uno excluyente del tipo "o esto o lo otro".

Contraponer lo centrado en el aprendizaje con lo centrado en la selección. Esta idea central se ha descripto al principio en este texto. Y constituye una idea recurrente: Todas las siguientes ideas básicas se establecen para apoyar esta idea central.

Contraponer la instrucción centrada en el alumno con la instrucción centrada en el docente. McCombs y Whisler (1997) definen la expresión "centrado en el estudiante" como:

La perspectiva que empareja un enfoque en los estudiantes individualmente (lo que asume de otros, las experiencias propias, perspectivas, antecedentes, talentos, intereses, capacidades y necesidades), con un enfoque en el aprendizaje (el mejor conocimiento disponible sobre el aprendizaje y cómo se produce, y sobre las prácticas docentes que son más eficaces para promover los más altos niveles de motivación, de aprendizaje y de logro para todos los alumnos). (p. 9)

A esto yo añadiría que los métodos de enseñanza son en gran parte a medida para cada alumno, y llevada a cabo por el alumno, en vez de por el profesor. Los estudiantes también juegan un papel más importante en la dirección de su propio aprendizaje, incluyendo la reflexión sobre él y de él.

Contraponer "aprender haciendo" contra aprender a través de las presentaciones del docente. La mayor parte del tiempo del estudiante se debe ocupar en la realización de tareas auténticas ${ }^{1}$, en lugar de escuchar a un docente. Algunos entienden ese tipo de Aprendizaje por Tareas en términos de entender al "estudiante como trabajador" y al "profesor como director", en lugar de entender al docente como trabajador (Schlechty, 2002). Otros entienden esto, el papel del maestro, como "el guía que acompaña" en lugar de "el sabio en la tarima". Algunos lo llaman el enfoque constructivista del aprendizaje. La conclusión es que el Aprendizaje por Tareas es activo, centrado en el alumno, y en gran medida auto-dirigido.

Contraponer progreso basado en logros con progreso basado en tiempo. Cada estudiante avanza a un nuevo tema o competencia cuando ha alcanzado un nivel de logro, y no cuando un determinado intervalo de tiempo ha pasado. Por tanto un estudiante no se ve obligado a avanzar antes de alcanzar el estándar, para ese tema o para esa competencia, y se le permite avanzar tan pronto como el estándar se alcanza. Esto evita la enorme pérdida de tiempo de los estudiantes que existe en el paradigma de la educación de la era industrial. Este es un enfoque basado en estándares para la educación en el sentido más verdadero de la palabra. El Aprendizaje por Dominios

\footnotetext{
${ }^{1}$ Entre las tareas auténticas se incluyen las basadas en problemas, en proyectos, en temas, en casos y en preguntas, todas las cuales son tipos de aprendizaje basado en el desempeño o formas de aprender haciendo.
} 
(Block, 1971; Bloom, 1968, 1981) fue una implementación temprana de esta idea central.

Contraponer la instrucción personalizada con la estandarizada. El nuevo paradigma ofrece experiencias de aprendizaje personalizadas en lugar de estandarizadas. Esto va más allá del progreso basado en logros (en el cual se ha personalizado el ritmo) para incluir contenido personalizado y métodos personalizados. Si bien hay un núcleo de conocimientos, habilidades y actitudes que todos los estudiantes aprenden, hay un tiempo considerable para que los estudiantes cultiven sus talentos, intereses y fortalezas particulares. Por otra parte, Howard Gardner ha demostrado que los estudiantes difieren en su perfil según los siete principales tipos de inteligencia y ha argumentado que las inteligencias más fuertes de un estudiante pueden ser utilizadas más eficazmente como "puntos de entrada" para el aprendizaje del conocimiento, habilidades y actitudes (Gardner, 1999). Los métodos también se personalizan para otros tipos de características del alumno y sus preferencias. Los Planes Personales de aprendizaje (diferentes en aspectos importantes de los $\mathrm{IEPs}^{2}$ ) y los contratos de aprendizaje son herramientas valiosas para personalizar el aprendizaje.

Contraponer la evaluación con referencia a criterios con la evaluación con referencia a normas. Los dos fines de la evaluación de los alumnos en el nuevo paradigma son guiar el aprendizaje del estudiante (evaluación formativa) y certificar los logros de estudiantes (evaluación sumativa). La evaluación normativa (otra forma de evaluación sumativa) ya no se utiliza. La evaluación formativa supone proporcionar a cada estudiante una respuesta inmediata sobre su rendimiento, con pistas u otras formas de orientación para ayudar al estudiante a aprender de los errores. La evaluación sumativa implica certificación cuando un estudiante ha alcanzado el estándar en la consecución establecida para un objetivo determinado.

Colaboración y trabajo individual. En los lugares de trabajo, la mayor parte del trabajo intelectual se hace en equipo. La colaboración es importante en la vida laboral, la vida cívica y la vida familiar. Por lo tanto, los estudiantes necesitan experiencias en la colaboración en equipos pequeños. El aprendizaje basado en tareas en equipos no sólo brinda una excelente oportunidad para que los estudiantes desarrollen sus habilidades de colaboración, sino que también proporciona una valiosa oportunidad para que aprendan unos de los otros. Por otra parte, esto se basa fuertemente en el constructivismo social (Palincsar, 1998 ; Scardemalia y Bereiter, 1996).

Lo agradable y lo desagradable. En la era del trabajo intelectual, el aprendizaje permanente es esencial para la calidad de vida de nuestros ciudadanos y para la salud de nuestras comunidades. El aprendizaje permanente se ve realzado por el deseo de aprender. El paradigma de educación de la era industrial hace que a muchos estudiantes no les guste aprender, y ha convertido a la cultura de nuestras escuelas en una cultura en la cual se devalúa y ridiculiza a los estudiantes que se destacan en el aprendizaje. Esa forma de pensar y esa cultura atenta contra el aprendizaje continuo. Aunque el aprendizaje permanente ha sido desde hace muchos años una palabra de moda en

\footnotetext{
${ }^{2}$ Los Planes Educativos Individualizados o Programas Educativos Individualizados se usan principalmente en la educación especial. En España se suelen llamar Adaptaciones Curriculares. Esta idea, la de los Planes Personales de aprendizaje (en lo sucesivo PPA), entendemos es central en el desarrollo teórico que hace el autor y seguramente tendrá un importante repercusión. No queda, en nuestro contexto, tan claro el concepto contrato de aprendizaje (Nota del Adaptador).
} 
educación, el paradigma de la era industrial de hecho lo impide. El paradigma postindustrial cambia esta relación al promover el gusto por el aprendizaje en los estudiantes. Para ello es necesario cambiar de motivación extrínseca a la motivación intrínseca. También se demanda que el aprendizaje se produzca a través de tareas auténticas y atractivas, como se suele hacer en el aprendizaje basado en problemas y en el aprendizaje basado en proyectos.

Éstas que hemos visto son ideas centrales que representan las características esenciales de los sistemas educativos y de formación post-industriales. Ideas que tienen un nivel de universalidad para la instrucción post-industrial de igual forma a como los primeros principios de Merrill son válidos para todos los paradigmas de la enseñanza. Sin embargo, las formas en que se lleven a cabo probablemente varíen considerablemente de un sistema educativo a otro. La siguiente es una visión de la instrucción para una posible implementación de estas ideas centrales.

\section{Instrucción basada en Tareas}

El compromiso o la motivación del estudiante es clave para el aprendizaje. No importa cuánto trabajo haga el profesor, si el estudiante no trabaja no aprende. La calidad y cantidad de aprendizaje son directamente proporcionales a la cantidad de esfuerzo que el estudiante dedica al aprendizaje. El paradigma de educación y formación de la era industrial se basa en la motivación extrínseca, con calificaciones, salas de estudio, quedarse sin recreo o retrasar la salida, y en el peor de los casos repitiendo un grado o reprobando.

Por el contrario, por varias razones, la motivación intrínseca adquiere especial importancia en el paradigma de la era de la información. Entre las razones se incluyen la importancia del aprendizaje permanente y, por lo tanto, del desarrollo de la estima por el aprendizaje, el deterioro de la disciplina en el hogar y en la escuela, y la menor eficacia de los motivadores extrínsecos actuales, que hemos visto, en relación a lo que sucedía 30 años atrás.

Para aumentar la motivación intrínseca, los métodos de enseñanza deben estar centrados en el alumno en lugar de en el profesor. Deben incluir el "aprender haciendo". Es decir deben utilizar las tareas que son de interés para el estudiante (esto por lo general significa que deben ser "auténticas"), y deben ofrecer oportunidades de colaboración. Esto hace que la instrucción basada en Tareas ${ }^{3}$ (en inglés:TBI-Task Based Instruction) sea particularmente apropiada como una teoría educativa fundamental dentro del paradigma de educación y de formación en la era de la información.

Por otra parte, dado que la importancia del progreso del estudiante se basa en el aprendizaje más que en el tiempo, los estudiantes progresan a ritmos diferentes y aprenden cosas diferentes en un momento dado. Este hecho también se presta bien a la TBI, ya que se dirige más al alumno que al docente.

\footnotetext{
3 Uso el término "instrucción basada en tareas" en vez de "aprendizaje por tareas" porque esto último es lo que el alumno hace, mientras que lo primero es lo que el docente o el sistema instruccional hace para apoyar el aprendizaje. Aún más, utilizo el término instrucción basada en tareas en sentido amplio para incluir a la instrucción por proyectos, por problemas, por temas, por caso y el aprendizaje basado en preguntas.
} 
Parece claro pues que la TBI debe tener un lugar destacado en el nuevo paradigma de la educación y de la formación. Sin embargo existen problemas con esta modalidad de instrucción, que son los que exploro en el siguiente punto.

\section{Problemas con la Instrucción basada en Tareas}

En mi propio uso de la TBI, me he encontrado con cuatro problemas significativos. La mayoría de la educación basada en tareas es colaborativa, o se hace con trabajo en equipo, y en general todo el equipo se evalúa según un producto final. Esto hace que sea difícil de evaluar y que no se pueda asegurar que todos los estudiantes han aprendido lo que tenían que aprender o lo que se quería que aprendieran. He encontrado también que a menudo uno de los estudiantes en el equipo es haragán y no aprendía mucho. También he encontrado que los integrantes del grupo suelen trabajar de forma cooperativa en vez de colaborativa, lo que significa que cada uno realiza tareas diferentes y por lo tanto aprende cosas diferentes. En mi experiencia, es raro que algún estudiante haya aprendido todo lo que se pretendía. Para un sistema en el cual el progreso del alumno se basa en el aprendizaje, es importante evaluar y garantizar lo que aprende cada alumno en el equipo. Sin embargo, es raro que esto suceda en la TBI. Esto puede ser un problema no tan difundido en los niveles superiores de educación, pero es un gran problema en los niveles inferiores, debido a que las deficiencias en el aprendizaje pueden hacer que los aprendizajes relacionados resulten difíciles y resulten frustrantes en el futuro.

En segundo lugar, las habilidades y competencias que enseñamos a través de la TIB son generalmente aquellas que nuestros alumnos tendrán que transferir a una amplia gama de situaciones, sobre todo para tareas cognitivas complejas. Sin embargo, en la TBI los estudiantes suelen utilizar una habilidad sólo una vez o dos veces en la ejecución del proyecto. Esto hace que sea difícil para ellos aprender a utilizar la destreza en toda la gama de situaciones en las que es probable que sea necesaria en el futuro. Muchas habilidades requieren mucha práctica para desarrollarlas a un nivel de competencia o de experticia; sin embargo, esto rara vez ocurre en TBI.

En tercer lugar, es necesario automatizar algunas habilidades con el fin de liberar a la persona de los procesos cognitivos conscientes de pensamiento de alto nivel requeridos durante la ejecución de una tarea. La TBI no responde a esta necesidad de instrucción.

Por último, se puede perder mucho tiempo de aprendizaje en la TBI - buscando información, realizando actividades improductivas, repitiendo el uso de las habilidades que ya han sido dominados, y esforzarse por aprender sin la suficiente orientación o apoyo. A menudo es importante, no sólo en la formación de empresa, sino también en la educación básica y en la educación superior, obtener el mayor aprendizaje en el menor tiempo posible. Esta eficacia no suele ser una característica típica de la TBI.

Teniendo en cuenta estos cuatro problemas con laTBI - la dificultad para asegurar el dominio, la transferencia, la automatización, y la eficiencia. ¿Significa esto que deberíamos abandonar la TBI y aplicar la instrucción directa, como Kirschner, Sweller y Clark (2006) proponen? Para citar un famoso anuncio, "no exactamente". Ahora profundizaré este tema. 


\section{Una visión del paradigma post-industrial de "Instrucción por tareas y espacios instruccionales"}

Imagine un pequeño equipo de estudiantes que trabajan en una labor auténtica en una simulación con un ordenador (el "espacio de la tarea"). Pronto se encuentran con una brecha en el aprendizaje (conocimientos, habilidades, comprensiones, valores, actitudes, disposiciones, etc.) que necesitan que desaparezca para continuar con la tarea. Imaginemos que los estudiantes pueden "congelar" el tiempo y tener un mentor virtual que aparece y proporciona una tutoría personalizada "justo a tiempo" para desarrollar esa habilidad o conocimiento individual en cada estudiante (o sea: el "espacio instruccional").

La investigación muestra que el aprendizaje de una habilidad se facilita en la medida en que la instrucción le diga a los estudiantes cómo hacerlo, les muestre cómo hacerlo en diversas situaciones, y les dé la práctica con retroalimentación inmediata, una vez más en diversas situaciones (Merrill, 1983; Merrill, Reigeluth, y Faust, 1979), por lo que los estudiantes aprenden a generalizar o a transferir la habilidad para toda la gama de situaciones que encontrarán en el mundo real. Cada estudiante continúa practicando hasta que alcanza el nivel de dominio de la habilidad, como ocurre en la Academia Khan (www.khanacademy.com). Al llegar al estándar requerido, el estudiante regresa al espacio de la tarea, y el tiempo se descongela para aplicar lo aprendido a la tarea y seguir trabajando en ella hasta que se encuentra la siguiente brecha de aprendizaje, y así el ciclo hacer-aprender-hacer se repite.

En la actualidad se han desarrollado teorías instruccionales bien validadas para ofrecer una guía para el diseño, tanto del espacio de la tarea como para el espacio de instrucción (ver Reigeluth, 1999b; Reigeluth y Carr Chellman, 2009c, por ejemplo). De esta manera, trascendemos el pensamiento disyuntivo - "uno u otro" -, tan característico de la era industrial, para pasar al pensamiento conjuntivo o aditivo - "ambos/y" -, que se adapta mejor a la complejidad, mucho mayor, inherente a la era de la información. Utilizamos pues una teoría instruccional que combina lo mejor de las teorías y modelos conductistas, cognitivistas y constructivistas. Esta teoría presta especial atención al dominio de las competencias individuales, pero también evita la fragmentación característica de muchos de los programas que pretendían explicar el aprendizaje en el pasado.

\section{Evaluación en equipo y evaluación individual}

Siguiendo con los problemas de la TBI, particularmente de la forma en que a menudo se implementa, tenemos el originado porque los estudiantes son evaluados según la calidad del "producto" del equipo. Esto no da la menor idea de quién ha adquirido cuáles competencias. Asimismo, no da ninguna indicación acerca de la capacidad de cada estudiante para transferir esas competencias a otras situaciones donde puedan ser necesarias. La evaluación de equipo es importante, pero también se necesita una evaluación individual, y el espacio instruccional ofrece una excelente oportunidad para satisfacer esta necesidad. Al igual que el espacio de la tarea, el espacio instruccional está orientado al desempeño. Las oportunidades de práctica (ofrecidas principalmente a través de una simulación por ordenador para tener una respuesta inmediata, personalizada y autenténtica) continúan siendo ofrecidas al estudiante hasta que éste alcanza el criterio de un determinado número de aciertos continuos que sean requeridos 
por la norma. La evaluación formativa se comunica inmediatamente a los estudiantes en cada actuación incorrecta, a menudo en forma de consejos que promueven el procesamiento cognitivo más profundo y la comprensión. Cuando la automatización de una habilidad (Anderson, 1996) es importante, también hay un criterio sobre la velocidad de rendimiento que se debe cumplir.

De esta manera, la evaluación del estudiante se integra plenamente en la instrucción, y no hay pérdida de tiempo en la realización de una evaluación por separado. Además, la evaluación asegura que cada estudiante ha alcanzado el estándar para toda la gama de situaciones en las esa competencia sean necesarias.

Cuando una ejecución no puede llevarse a cabo a través de un ordenador (por ejemplo, una función de ballet), un experto tiene un dispositivo móvil con una rúbrica para la evaluación. El experto completa la rúbrica mientras observa la ejecución, y ofrece evaluación formativa cuando sea apropiado durante su transcurso, esto permite que el estudiante lo vuelva a intentar cuando su rendimiento sea inferior al nivel estándar y entonces realizar una nueva evaluación e introducir la información automáticamente en el sistema informático, la cual se almacena en el registro del estudiante. El estudiante u otras personas autorizadas pueden acceder al registro para ver el resultado de esa evaluación.

\section{Teoría Instruccional para el Espacio de la Tarea}

No hay muchas orientaciones validadas para el diseño del espacio de la tarea. Pero entre ellas se encuentran los principios universales y situados para el espacio de la tarea (véase, por ejemplo, Barrows, 1986; Barrows y Tamblyn, 1980; Duffy y Raymer, 2010; Jonassen, 1997, 1999; Savery, 2009). Estos trabajos incluyen orientaciones sobre cómo seleccionar una buena tarea con el nivel de complejidad adecuado, formar pequeños grupos, autodirigir el aprendizaje, sobre lo que el docente debe hacer, cómo interrogar, y mucho más. Las simulaciones por ordenador suelen ser muy eficaces para crear y alojar el entorno de trabajo, pero el espacio de la tarea podría estar compuesto enteramente de lugares, objetos y personas en el mundo real (aprendizaje basado en el lugar), o podría ser una combinación de simulación por ordenador y entornos del mundo real. STAR LEGACY (Schwartz, Lin, Brophy, y Bransford, 1999) es un buen ejemplo de una simulación por ordenador basada para el espacio de proyecto.

\section{Teoría instruccional para el espacio instruccional}

La selección de estrategias de enseñanza en el espacio instruccional se basa principalmente en el tipo de aprendizaje (fines de la instrucción) de que se trate (véase la Unidad 3 en Reigeluth y Carr Chellman, 2009c). Para la memorización, resultan especialmente eficaces la ejercitación mecánica y la práctica (Salisbury, 1990), incluida la división en partes, la repetición, la inducción, y la mnemotecnia. Para aplicaciones (habilidades de aplicación), son muy eficaces los tutoriales de carácter general, los ejemplos, la práctica y la retroalimentación inmediata (Merrill, 1983; Romiszowski, 2009). Para la comprensión conceptual, la conexión de nuevos conceptos con los conceptos existentes en las estructuras cognitivas del estudiante requiere el uso de métodos tales como analogías, el contexto (y dentro de él los organizadores previos), la comparación y el contraste, el análisis de las partess y los tipos, y otras técnicas diversas basadas en las dimensiones de la comprensión requerida (Reigeluth, 1983). Para una mejor comprensión teórica, las relaciones causales se aprenden mejor a través de explorar las causas (la explicación), los efectos (predicción), y las soluciones (solución de problemas). Los procesos naturales se aprenden mejor a través de la descripción de la 
secuencia de eventos en el proceso natural propio (Reigeluth y Schwartz, 1989). Este tipo de estrategias de enseñanza ha sido bien investigado para determinar su eficacia, su eficiencia, y la aceptación. Y a menudo se implementan mejor a través de tutoriales, simulaciones y juegos de ordenador.

De nuevo, esto no es más que una visión del paradigma post-industrial de la instrucción. Animo al lector a tratar de pensar en visiones adicionales que satisfagan las necesidades de la era post-industrial: principalmente la motivación intrínseca, la personalización, el progreso basado en el nivel de logro del estudiante, el aprendizaje colaborativo y el aprendizaje autodirigido. Para ello, puede ser útil tener en cuenta las formas en que los roles son propensos a cambiar en el nuevo paradigma de la enseñanza.

\section{Roles claves en el paradigma post-industrial de Instrucción ${ }^{4}$}

Es probable que los roles de los profesores, de los estudiantes, y de la tecnología sufran cambios. Cada uno de estos tipos roles se describe brevemente a continuación.

\section{Nuevos roles para los docentes}

El rol del profesor ha cambiado drásticamente en el nuevo paradigma de enseñanza al pasar de ser el "sabio en la tarima" a ser el "guía que acompaña". Actualmente, distingo tres funciones principales inherentes al rol de guía. En primer lugar, el profesor es un diseñador de trabajo de los estudiantes (Schlechty, 2002). El trabajo de los estudiantes incluye lo que se hace tanto en el espacio de la tarea como en el espacio instruccional. En segundo lugar, el profesor es un facilitador del proceso de aprendizaje. Esto incluye ayudar a desarrollar un plan de aprendizaje personal, entrenar o ayudar a crear andamios para el aprendizaje del alumno cuando sea apropiado, facilitar el debate y la reflexión, y organizar la disponibilidad de recursos diversos, humanos y materiales. En tercer lugar, y quizás lo más importante en el sector de la educación pública, el profesor es un mentor atento, una persona que se ocupa del desarrollo integral, pleno y de calidad del estudiante.

Pero las facetas del profesor como diseñador, como facilitador y como mentor, son sólo tres de los nuevos roles, los más importantes, que los docentes han de desarrollar, pero no todos los profesores han de desempeñar todos los roles. Los diferentes tipos de profesores con diferentes tipos y niveles de formación y experiencia puede centrarse en uno o dos de estos papeles (incluyendo a los estudiantes en el papel de profesores véase la sección siguiente).

\section{Nuevos roles de los estudiantes}

En primer lugar, el aprendizaje es un proceso activo. El estudiante debe hacer un esfuerzo para aprender. El maestro no lo puede hacer por el estudiante. Esta es la razón por la cual Schlechty (2002) caracteriza al nuevo paradigma como aquel en el cual el estudiante es el trabajador, no el maestro, y en el cual el maestro es el diseñador del trabajo del estudiante.

\footnotetext{
${ }^{4}$ Gran parte de esta sección está basada en Reigeluth (2009) y en Reigeluth, Watson, S., Watson, W., Dutta, Chen y Powell (2008).
} 
En segundo lugar, para preparar al estudiante para el aprendizaje permanente, el maestro ayuda a cada estudiante a convertirse en un estudiante autodirigido y motivado. Los estudiantes están motivados para aprender desde que nacen hasta que ingresan a la escuela por primera vez. El paradigma del cambio de etapa destruye sistemáticamente la motivación mediante la eliminación de todas las decisiones propias y al asignarle a los estudiantes tareas aburridas que no son relevantes para sus vidas. En contraste, el sistema post-industrial está diseñado para fomentar la auto-motivación a través de la posibilidad de que el estudiante se dirija a sí mismo y a través del aprendizaje activo en el contexto de las tareas pertinentes, interesantes. La motivación del estudiante es clave para la productividad de la educación y para ayudar a los estudiantes a darse cuenta de su potencial. También reduce en gran medida los problemas de disciplina, el uso de drogas (sic), y mucho más.

En tercer lugar, se dice a menudo que la mejor manera de aprender algo es enseñándolo. Los estudiantes son tal vez el recurso más infrautilizado en nuestros sistemas escolares. Por otra parte, alguien que acaba de aprender algo es a menudo mejor para ayudar a aprender a otro, en vez de quien lo aprendió hace mucho tiempo. Además de los estudiantes de más edad, que enseñan a lo que son algo más jóvenes que ellos, los pares pueden aprender unos de otros a través de proyectos de colaboración. Los alumnos también pueden actuar como tutores.

Por lo tanto, los nuevos roles de los estudiantes incluyen al estudiante como trabajador, como estudiante autónomo, y como profesor.

\section{Nuevos roles para la tecnología}

Actualmente veo cuatro funciones principales de la tecnología para hacer que el nuevo paradigma de instrucción sea viable y rentable (Reigeluth y Carr Chellman, 2009c; Reigeluth et al, 2008). Cada una de ellas se describe a continuación. Son sustanciales para el sector de la educación pública básica, pero estos roles son igualmente relevantes en la educación superior, en la capacitación corporativa, en el entrenamiento militar, y en la educación y la formación en otros contextos.

Registro del aprendizaje de los estudiantes. El seguimiento del progreso de los estudiantes según el avance en sus logros requiere de un registro individual de ese seguimiento. La tecnología ahorra al profesor mucho tiempo en esa tarea. En esta función, la tecnología sustituye al boletín de calificaciones actual. Se compone de tres partes. En primer lugar, tiene un inventario de las normas que incluye tanto los estándares de educación requeridos (nacional, estatal y local) y a los estándares educativos opcionales para el acceso por parte del profesor, del estudiante, y de los padres. Estas normas se descomponen en logros individuales y se muestran en una "mapa de dominio" de formato similar al de la Academia Khan. La Teoría de dominio (Bunderson, Wiley, y McBride, 2009) es muy útil para formular esta herramienta tecnológica. Presenta una lista de logros que deben o pueden ser alcanzados, junto con los niveles o estándares o criterios en el que cada uno se puede o se debe alcanzar. En segundo lugar, tiene un inventario de logros personales que contiene un registro de lo que cada estudiante sabe. En esencia, describe el progreso de cada estudiante en relación a los logros que figuran en el inventario de las normas (y tal vez algunos de los que todavía no estén listados allí). Muestra: cuándo se ha alcanzado un determinado logro, cuáles son los logros requeridos, cuál es la meta siguiente a alcanzar en cada área, y los enlaces a la evidencia de cada logro (bajo la forma de un resumen de datos y/o 
objeto patrón original). En tercer lugar, el Inventario de Carácterísticas Personales ${ }^{5}$ que registra las características personales de cada estudiante; aquellas que influyen su aprendizaje tales como su estilo de aprendizaje, su perfil de inteligencias múltiples, sus necesidades especiales, sus intereses y objetivos y los eventos más importantes en su vida ( Reigeluth \& Carr-Chellman, 2009c ; Reigeluth et al., 2008) .

La planificación para el aprendizaje del estudiante. El plan personal de aprendizaje, o contrato $^{6}$, puede resultar complejo para que los docentes lo desarrollen para todos sus estudiantes. Aquí, una vez más, surge un rol para el cual la tecnología tiene características ideales. Puede ayudar a que el estudiante, los padres y el docente a) decidan los objetivos a largo plazo, b) identifiquen la gama completa de opciones de metas que están al alcance de los estudiantes; c) seleccionen de entre esas opciones aquellas a las cuales el estudiante desea abocarse (objetivos a corto plazo), basándose en los requisitos, los objetivos a largo plazo, sus intereses, las oportunidades, etc.; d) identificar o crear tareas para lograr esos objetivos a corto plazo, e) identificar y combinar con otros estudiantes que estén interesados en realizar las mismas tareas al mismo tiempo (si se desea o necesita colaboración) y específicamente los roles de todos los compañeros de equipo, f) especificar los roles que el profesor, padre y cualquier otro mentor deba cumplir con relación a apoyar al estudiante a aprender a partir de la tarea, y g) desarrollar un contrato que especifique las metas, tareas, equipos, roles de los estudiantes y responsabilidades, roles de maestros y padres, método de evaluación y la fecha tope para cada tarea (Reigeluth et al., 2008).

Instrucción para el aprendizaje del alumno. Tratar de "instruir" a 25 alumnos que están aprendiendo distintas cosas al mismo tiempo puede ser muy difícil para el docentes, sobre todo si tiene que ser el agente instruccional todo el tiempo, tal como es típico en el paradigma de la era industrial. Sin embargo, la tecnología puede mostrar al estudiante (o al pequeño grupo) la introducción a la tarea en el espacio de la tarea, poner a disposición las herramientas instruccionales (como pueden ser simulaciones, tutoriales, ejercicios mecánicos y de práctica, herramientas para la investigación, para la comunicación y objetos de aprendizaje) en el espacio instruccional, para apoyar el aprendizaje durante la tarea (tal como se describió anteriormente), brindar herramientas para el monitoreo y el apoyo al progreso en la realización de la tarea, y aún más, poner a disposición herramientas para ayudar a que los docentes y otros desarrollen nuevas tareas y herramientas instruccionales. La tecnología puede poner a disposición de los alumnos todas las funciones antes mencionadas en cualquier tiempo y lugar. La teoría instruccional es extremadamente importante para guiar el diseño de estas herramientas (Reigeluth et al., 2008).

Evaluación para y del aprendizaje del alumno. Otra vez más, en este caso, llevar a cabo evaluaciones formativas y sumativas podría resultar una pesadilla para los docentes, ya que los estudiantes no realizan todos una prueba determinada al mismo

\footnotetext{
${ }^{5}$ Nota del adaptador: El concepto Inventario de Carácterísticas Personales parece ser por la definición muy próximo al que en otros contextos se ha llamado Identidad de Aprendizaje en Red o Identidad Social de Aprendizaje solo que en ese caso hace referencia a la tecnología en general (local o en red) y en aquel a las herramientas de web social.

${ }^{6}$ Nota del adaptador: En la ocasión anterior que aparecía el término contrato no figuraba como estrictamente identificado con el plan personal de aprendizaje. Ahora sí. Por tanto consideraremos que responden a un mismo instrumento de seguimiento del aprendizaje individual.
} 
tiempo. Y una vez más, la tecnología puede ofrecer un gran alivio. En primer lugar, tal como se mencionó anteriormente, la evaluación está integrada con el aprendizaje. La enorme cantidad de oportunidades de ejecución se usan tanto en las evaluaciones formativas como en las sumativas. En segundo lugar, las evaluaciones se presentan como tareas auténticas en las cuales los estudiantes demuestran sus conocimientos, comprensión y habilidad. En tercer lugar, ya sea en una simulación, o en un tutorial, o en una ejercitación mecánica o práctica, la tecnología está diseñada para evaluar si se cumplió o no con el criterio de evaluación en cada desempeño del estudiante, y para brindar una devolución formativa al estudiante en forma inmediata para obtener un mayor impacto. Cuando los criterios que indican una ejecución exitosa se han cumplido en una cantidad $\boldsymbol{x}$ de veces de las $\boldsymbol{y}$ últimas ejecuciones, se considera que se completa la evaluación sumativa y el logro correspondiente queda asentado automáticamente en el inventario personal de logros del estudiante. En unos pocos casos, en los cuales la tecnología no puede evaluar el desempeño, un observador mediante un dispositivo móvil que contiene una rúbrica para la evaluación, brinda personalmente la devolución inmediata sobre la ejecución del alumno. La información proveniente del dispositivo móvil se carga en el sistema informático en el cual esté alojado el inventario personal del estudiante. Finalmente, la tecnología brinda herramientas para ayudar a que los profesores desarrollen evaluaciones y los enlacen a los estándares (Reigeluth et al., 2008).

Nótese que estos cuatro roles o funciones están totalmente integrados (Reigeluth \& Carr-Chellman, 2009c). La herramienta que almacena los registros brinda información automáticamente para la herrramienta de planificación. La herramienta de planificación identifica las herramientas instruccionales que están disponibles. La herramienta de evaluación está integrada en la herramienta instruccional. Y la herramienta de evaluación alimenta con información a la herramienta de almacenamiento de registros automáticamente (Reigeluth et al., 2008 ; Watson, Lee, \& Reigeluth, 2007). En nuestro trabajo anterior utilizamos el término "Sistema de Gestión de Aprendizaje" (Learning Management System, en inglés) para esta herramienta integral, personalizada y exhaustiva, pero ese término se usa habitualmente para describir sistemas de gestión de cursos centrados en el docente. Es por eso que, para evitar confusiones, hemos decidido llamarlos Sistemas Personalizados de Educación Integral (en inglés, Personalized Integrated Educational System -PIES).

De igual modo, nótese que hay muchos roles más en los PIES (Reigeluth et al., 2008). Entre estos roles "secundarios" se incluyen las comunicaciones (correo electrónico, blogs, sitios web, foros de discusión, wikis, pizarras interactivas, mensajería instantánea, podcasts, videocasts, etc.), la administración de PIES (que ofrecen acceso a la información y la autoridad para agregar información según el tipo de que se trate y el rol); la administración de datos generales sobre los estudiantes (su dirección, información sobre los padres y/o tutores, de su mentor/profesor-tutor y escuela, ubicación de la escuela y asistencia, información de salud, etc.), la información sobre el personal de la escuela (dirección, acreditaciones, función y empleos, ubicación, estudiantes asignados, herramientas creadas, evaluaciones de estudiantes realizadas, plan de desarrollo profesional del docente y su registro, repositorio de herramientas de enseñanza, premios que sus alumnos hayan recibido), y más.

Debería estar claro que la tecnología cumplirá un rol crucial en el éxito de la educación en el paradigma post-industrial. La tecnología posibilitará un salto espectacular en la mejora del aprendizaje, y posiblemente a un menor costo por estudiante por año que en 
el paradigma de la era industrial actual. Del mismo modo que la hoja de cálculo electrónica ha vuelto el trabajo del contador más rápido, fácil, económico y placentero, los PIES aquí descriptos harán que el trabajo docente sea más rápido, fácil, enconómico y plancentero. Pero la teoría instruccional es de imperiosa necesidad para que la teconología realice su contribución en todo su potencial.

\section{Conclusión}

En el mundo post-industrial, necesitamos transformar la mayoría de nuestros sistemas de educación y de capacitación, cambiarlos desde aquellos que fueron diseñados para seleccionar a los alumnos, a otros que estén diseñados para maximizar el aprendizaje -, desde aquellos en los cuales el progreso de los alumnos se mide en función del tiempo a otros basados en sus logros. Esta transformación requerirá de avances tanto en la teoría como en la tecnología instruccional.

Los principios de Merril señalados al principio (centralidad de la tarea, activación, demostración, aplicación e integración) ofrecen un buen resumen, aunque general, de las características más importantes para una instrucción de alta calidad. Para una orientación más detallada, debemos observar las "situaciones" que determinan las formas en las cuales la instrucción debe diferir de unos casos a otros. Las investigaciones llevadas a cabo hasta el momento indican que estas distintas situaciones basan su variedad en los distintos medios (distintos enfoques de instrucción), y en las diferentes finalidades (distintos resultados de la formación o tipos de aprendizaje).

Además, resulta útil tener una visión más holística de cómo sería un nuevo paradigma de instrucción. Propongo que se caracterice por las siguientes ideas centrales: centrado en el aprendizaje, centrado en el alumno, aprender haciendo, avance basado en logros, instrucción personalizada, evaluación basada en criterios, aprendizaje colaborativo y aprendizaje placentero. Propongo, entonces, una visión de la instrucción para una implementación posible de estas ideas centrales, la cual incluye espacios de tareas e instruccionales, evaluación grupal e individual, estrategias instruccionales para el espacio de tareas, y estrategias instruccionales para el espacio instruccional.

Finalmente, resumo un conjunto de roles claves para el nuevo paradigma de instrucción. Entre los nuevos roles para los docentes se incluyen: diseñador del trabajo del alumno, facilitador del proceso de aprendizaje y mentor atento. Entre los nuevos roles para los estudiantes se incluyen: trabajador, estudiante autónomo y co-instructor. Se describieron cuatro nuevos roles centrales para la tecnología. En primer lugar, el almacenamiento de los registros de aprendizaje de los alumnos, que incluye ofrecer un inventario de estándares, un inventario de logros personales y un inventario de características personales. En segundo lugar, la planificación del aprendizaje del alumno, que incluye ayudar al estudiante, a sus padres y al docente a identificar o decidir los objetivos a largo plazo, las metas al alcance del alumno en el presente, las metas a alcanzar en el futuro inmediato, los otros estudiantes que puedan trabajar en el grupo, los roles del docente y de los padres y el plan personal de aprendizaje. En tercer lugar, la instrucción para el aprendizaje de los alumnos incluye una amplia variedad de herramientas tanto para el espacio de la tarea como para el espacio instruccional. Finalmente, la evaluación para y del aprendizaje del alumno está integrada con la instrucción, utiliza tareas y ejecuciones auténticas, certificaciones de logros y retroalimentación formativa. 
Aunque gran parte de la teoría instruccional se ha generado para guiar el diseño del nuevo paradigma de instrucción, aún queda mucho por aprender. Necesitamos aprender cómo responder mejor al fuerte componente emocional del aprendizaje (Greenspan, 1997), cómo promover el desarrollo social y emocional, y cómo promover el desarrollo de actitudes, valores, moral y ética positivos entre otras cosas. Tengo la esperanza de que usted, lector, recoja el guante en este desafío y colabore en el desarrollo de los conocimientos que necesitamos para mejorar aún más nuestra capacidad de ayudar a que cada alumno desarrolle todo su potencial.

Presentación del artículo en versión original: 20 de septiembre de 2012 Fecha de aprobación: 25 de septiembre de 2012 Fecha de publicación: 15 de julio de 2016

Reigeluth, C. (2016). Teoría instruccional y tecnología para el nuevo paradigma de la educación. RED. Revista de Educación a Distancia. Número 50.30 de septiembre de 2016. Consultado el (dd/mm/aaa) en http://www.um.es/ead/red/50

\section{Referencias}

Anderson, J. R. (1996). The architecture of cognition. Mahwah, NJ: Lawrence Erlbaum Associates.

Barrows, H. S. (1986). A taxonomy of problem-based learning methods. Medical Education, 20(6), 481-486. doi: 10.1111/j.1365-2923.1986.tb01386.x

Barrows, H. S., \& Tamblyn, R. M. (1980). Problem-based learning: An approach to medical education. New York: Springer.

Block, J. H. (1971). Mastery learning: Theory and practice. New York: Holt, Rinehart and Winston, Inc.

Bloom, B. S. (1968). Learning for mastery. Evaluation Comment, 1(1), 1-12.

Bloom, B. S. (1981). All our children learning. New York: McGraw-Hill.

Bunderson, C. V., Wiley, D. A., \& McBride, R. (2009). Domain Theory for instruction: Mapping attainments to enable learner-centered education. In C. M. Reigeluth \& A. A. Carr-Chellman (Eds.), Instructional-design theories and models: Building a common knowledge base (Vol. III, pp. 327-347). New York: Routledge.

Duffy, T. M., \& Raymer, P. L. (2010). A practical guide and a constructivist rationale for inquiry based learning. Educational Technology, 50(4), 3-15.

Gardner, H. E. (1999). Multiple approaches to understanding. In C. M. Reigeluth (Ed.), Instructional-design theories and models, vol. II: A new paradigm of instructional theory (pp. 69-89). Mahwah, NJ: Lawrence Erlbaum Associates.

Greenspan, S. I. (1997). The growth of the mind and the endangered origins of intelligence. Reading, MA: Addison-Wesley Publishing Company.

Jonassen, D. H. (1997). Instructional design models for well-structured and illstructured problem-solving learning outcomes. Educational Technology Research and Development, 45(1), 65-94. 
Jonassen, D. H. (1999). Designing constructivist learning environments. In C. Reigeluth (Ed.), Instructional-Design Theories and Models (pp. 215-239). Mahwah, New Jersey: Lawrence Erlbaum.

Kirschner, P. A., Sweller, J., \& Clark, R. E. (2006). Why minimal guidance during instruction does not work: An analysis of the failure of constructivist, discovery, problem-based, experiential, and inquiry-based teaching. Educational Psychologist, 41(2), 75-86.

McCombs, B., \& Whisler, J. S. (1997). The learner-centered classroom and school: Strategies for increasing student motivation and achievement. San Francisco: Jossey-Bass Publishers.

Merrill, M. D. (1983). Component display theory. In C. M. Reigeluth (Ed.), Instructional-design theories and models: An overview of their current status. Hillsdale, NJ: Lawrence Erlbaum Associates.

Merrill, M. D. (2007). First principles of instruction: A synthesis. In R. A. Reiser \& J. V. Dempsey (Eds.), Trends and issues in instructional design and technology (2nd ed., pp. 62-71). Upper Saddle River, NJ: Merrill/Prentice-Hall.

Merrill, M. D. (2009). First principles of instruction. In C. M. Reigeluth \& A. A. CarrChellman (Eds.), Instructional-design theories and models: Building a common knowledge base (Vol. III, pp. 41-56). New York: Routledge.

Merrill, M. D., Reigeluth, C. M., \& Faust, G. W. (1979). The Instructional Quality Profile: A curriculum evaluation and design tool. In H. F. O'Neil, Jr. (Ed.), Procedures for Instructional Systems Development. New York: Academic Press.

Palincsar, A. S. (1998). Social constructivist perspectives on teaching and learning. Annual Review of Psychology, 49, 345-375.

Reigeluth, C. M. (1983). Meaningfulness and Instruction: Relating What Is Being Learned to What a Student Knows. Instructional Science, 12(3), 197-218.

Reigeluth, C. M. (1987). The search for meaningful reform: A third-wave educational system. Journal of Instructional Development, 10(4), 3-14.

Reigeluth, C. M. (1994). The imperative for systemic change. In C. M. Reigeluth \& R. J. Garfinkle (Eds.), Systemic change in education (pp. 3-11). Englewood Cliffs, NJ: Educational Technology Publications.

Reigeluth, C. M. (1999a). What is instructional-design theory and how is it changing? In C. M. Reigeluth (Ed.), Instructional-design theories and models: A new paradigm of instructional theory (Vol. II, pp. 5-29). Mahwah, NJ: Lawrence Erlbaum Associates.

Reigeluth, C. M. (2009). Instructional theory for education in the information age. In C. M. Reigeluth \& A. A. Carr-Chellman (Eds.), Instructional-design theories and models: Building a common knowledge base (Vol. III, pp. 387-399). New York: Routledge.

Reigeluth, C. M. (2011). An instructional theory for the post-industrial age. Educational Technology, 51(5), 25-29. 
Reigeluth, C. M. (2012). Instructional theory and technology for a post-industrial world. In R. A. Reiser \& J. V. Dempsey (Eds.), Trends and issues in instructional design and technology (3rd ed., pp. 75-83). Boston: Pearson Education.

Reigeluth, C. M. (Ed.). (1999b). Instructional-design theories and models: A new paradigm of instructional theory (Vol. II). Mahwah, N.J.: Lawrence Erlbaum Associates.

Reigeluth, C. M., \& Carr-Chellman, A. A. (2009a). Situational principles of instruction. In C. M. Reigeluth \& A. A. Carr-Chellman (Eds.), Instructional-design theories and models: Building a common knowledge base (Vol. III, pp. 57-68). New York: Routledge.

Reigeluth, C. M., \& Carr-Chellman, A. A. (2009b). Understanding instructional theory. In C. M. Reigeluth \& A. A. Carr-Chellman (Eds.), Instructional-design theories and models: Building a common knowledge base (Vol. III, pp. 3-26). New York: Routledge.

Reigeluth, C. M., \& Carr-Chellman, A. A. (Eds.). (2009c). Instructional-design theories and models: Building a common knowledge base (Vol. III). New York: Routledge.

Reigeluth, C. M., \& Garfinkle, R. J. (1994). Envisioning a new system of education. In C. M. Reigeluth \& R. J. Garfinkle (Eds.), Systemic Change in Education (pp. 5970). Englewood Cliffs, NJ: Educational Technology Publications.

Reigeluth, C. M., \& Schwartz, E. (1989). An instructional theory for the design of computer-based simulations. Journal of Computer-Based Instruction, 16(1), 1-10.

Reigeluth, C. M., Watson, S. L., Watson, W. R., Dutta, P., Chen, Z., \& Powell, N. (2008). Roles for technology in the information-age paradigm of education: Learning management systems. Educational Technology, 48(6), 32-39.

Romiszowski, A. (2009). Fostering skill development outcomes. In C. M. Reigeluth \& A. A. Carr-Chellman (Eds.), Instructional-design theories and models: Building a common knowledge base (Vol. III, pp. 199-224). New York: Routledge.

Salisbury, D. F. (1990). Cognitive psychology and Its implications for designing drill and practice programs for computers. Journal of Computer-Based Instruction, 17(1), 23-30.

Savery, J. R. (2009). Problem-based approach to instruction. In C. M. Reigeluth \& A. A. Carr-Chellman (Eds.), Instructional-design theories and models: Building a common knowledge base (Vol. III, pp. 143-165). New York: Routledge.

Scardemalia, M., \& Bereiter, C. (1996). Computer support for knowledge-building communities. In T. Koschmann (Ed.), CSCL: Theory and practice of an emerging paradigm (pp. 249-268). Mahwah, NJ: Lawrence Erlbaum Assoc.

Schlechty, P. (2002). Working on the work. New York: John Wiley \& Sons.

Schwartz, D. L., Lin, X., Brophy, S., \& Bransford, J. D. (1999). Toward the development of flexibly adaptive instructional designs. In C. M. Reigeluth (Ed.), Instructional-design theories and models: A new paradigm of instructional theory (Vol. II, pp. 183-213). Mahwah, NJ: Lawrence Erlbaum.

Watson, W. R., Lee, S. K., \& Reigeluth, C. M. (2007). Learning management systems: An overview and roadmap of the systemic application of computers to education. In 
F. M. Neto \& F. V. Brasileiro (Eds.), Advances in Computer-Supported Learning (pp. 66-96). Hershey, PA: Information Science Publishing. 